\title{
Helmet therapy in infants with positional skull deformation: randomised controlled trial
}

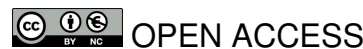

\author{
Renske M van Wijk $P h D$ candidate ${ }^{1}$, Leo $A$ van Vlimmeren senior researcher in paediatric \\ physiotherapy ${ }^{23}$, Catharina G M Groothuis-Oudshoorn biostatistician ${ }^{1}$, Catharina P B Van der Ploeg \\ epidemiologist ${ }^{4}$, Maarten J IJzerman professor ${ }^{1}$, Magda M Boere-Boonekamp associate professor \\ of youth health care ${ }^{1}$
}

${ }^{1}$ Department Health Technology and Services Research, Institute of Innovation and Governance Studies, University of Twente, Drienerlolaan 5, 7522 NB, Enschede, Netherlands; ${ }^{2}$ Department of Rehabilitation, Paediatric Physical Therapy, Radboud university medical center, Nijmegen, Netherlands; ${ }^{3}$ Scientific Institute for Quality of Healthcare, Radboud university medical center, Nijmegen, Netherlands; ${ }^{4} \mathrm{TNO}$ Child Health, Leiden, Netherlands

\begin{abstract}
Objective To determine the effectiveness of helmet therapy for positional skull deformation compared with the natural course of the condition in infants aged 5-6 months.

Design Pragmatic, single blinded, randomised controlled trial (HEADS, HElmet therapy Assessment in Deformed Skulls) nested in a prospective cohort study.

Setting 29 paediatric physiotherapy practices; helmet therapy was administered at four specialised centres.

Participants 84 infants aged 5 to 6 months with moderate to severe skull deformation, who were born after 36 weeks of gestation and had no muscular torticollis, craniosynostosis, or dysmorphic features. Participants were randomly assigned to helmet therapy $(n=42)$ or to natural course of the condition $(n=42)$ according to a randomisation plan with blocks of eight.

Interventions Six months of helmet therapy compared with the natural course of skull deformation. In both trial arms parents were asked to avoid any (additional) treatment for the skull deformation.

Main outcome measures The primary outcome was change in skull shape from baseline to 24 months of age assessed using plagiocephalometry (anthropometric measurement instrument). Change scores for plagiocephaly (oblique diameter difference index) and brachycephaly (cranioproportional index) were each included in an analysis of covariance, using baseline values as the covariate. Secondary outcomes were ear deviation, facial asymmetry, occipital lift, and motor development in the infant, quality of life (infant and parent measures), and parental satisfaction and anxiety. Baseline measurements were
\end{abstract}

performed in infants aged between 5 and 6 months, with follow-up measurements at 8,12 , and 24 months. Primary outcome assessment at 24 months was blinded.

Results The change score for both plagiocephaly and brachycephaly was equal between the helmet therapy and natural course groups, with a mean difference of -0.2 ( $95 \%$ confidence interval -1.6 to $1.2, P=0.80)$ and $0.2(-1.7$ to $2.2, P=0.81)$, respectively. Full recovery was achieved in 10 of 39 (26\%) participants in the helmet therapy group and 9 of 40 (23\%) participants in the natural course group (odds ratio $1.2,95 \%$ confidence interval 0.4 to $3.3, P=0.74$ ). All parents reported one or more side effects.

Conclusions Based on the equal effectiveness of helmet therapy and skull deformation following its natural course, high prevalence of side effects, and high costs associated with helmet therapy, we discourage the use of a helmet as a standard treatment for healthy infants with moderate to severe skull deformation.

Trial registration Current Controlled Trials ISRCTN18473161.

\section{Introduction}

Positional skull deformation is a condition in which the shape of an infant's skull deforms as a result of prolonged external forces. The infant's head is malleable and growing rapidly, hence it is susceptible to deformation, especially when infants develop a positional preference of the head when lying in the supine position. ${ }^{1}$ Two typical components of skull deformation are unilateral occipital flattening of the skull (plagiocephaly) and symmetrical occipital flattening (brachycephaly). ${ }^{2}$ A strong plagiocephalic flattening is often presented with ipsilateral 
frontal bossing of the forehead and anterior shift of the ipsilateral ear (ear deviation) and cheek (see web extra fig 1A). ${ }^{3-5}$

Brachycephaly can be accompanied by temporal bossing or an occipital lift (see web extra fig 1B). ${ }^{3}$

Skull deformation is generally considered a cosmetic condition. Developmental delays are regularly associated with skull deformation, ${ }^{6-9}$ but the deformation is increasingly seen as a marker for delays, instead of causing the delays.$^{6-8}$ Parents fear the negative physical and psychosocial effects of skull deformation on their child. ${ }^{10} 11$

The prevalence of skull deformation increased substantially after it was recommended that infants should be placed in a supine sleep position to prevent sudden infant death syndrome. ${ }^{12-18}$ Nowadays skull deformation seems most prevalent between two (16-22\%) and four months (20\%) after birth. ${ }^{19} 20$ The prevalence drops when infants become older. ${ }^{19-21}$

The preferred treatment is usually conservative. In a recently published guideline (2012), the Netherlands Centre of Preventive Child Health Care advised to start counselling parents during well baby visits on the handling and repositioning infants with an observed positional preference or skull deformation. When no improvement is seen at follow-up visits, infants are referred for paediatric physiotherapy. ${ }^{22}{ }^{23}$ Infants younger than 4 months may benefit from active repositioning, yet not all show improvement. ${ }^{22} 24$ As most infants show symmetry in posture at 5 or 6 months of age, ${ }^{122}$ no effects of continued paediatric physiotherapy can be expected. In infants with persistent skull deformation at 6 months of age, orthotic helmets or headbands are often prescribed. ${ }^{26-28} \mathrm{~A}$ helmet is a cranial orthosis made up of a rigid plastic shell with a foam lining. The helmet is expected to redirect skull growth by fitting closely to the infant's head but leaving room for the skull to grow at the flattened area. The helmet is recommended to be worn for 23 hours a day from 6 to 12 months of age. In the Netherlands $1-2 \%$ of infants (176 000 newborns in $2012^{29}$ ) received helmet therapy for skull deformation.

Since conclusive evidence from randomised trials is lacking, the clinical benefit of helmet therapy compared with the natural course of skull deformation remains unknown. The few prospective comparative studies to date tend to show positive results in favour of helmet therapy but have several limitations. ${ }^{50-33}$ Long term outcomes and assessment of side effects are missing in most studies and the clinical relevance of the reported effects is questionable. ${ }^{26} 283435$

The HElmet therapy Assessment in Deformed Skulls (HEADS) study is a randomised controlled trial designed to compare helmet therapy for six months with the natural course of positional skull deformation in infants aged 6 months. Although helmet therapy is expected to give slightly better results in the short term, we hypothesised that the natural course of skull deformation would catch up with the effects of helmet therapy over time and that no clinically meaningful differences would be present between the two groups at 2 years of age.

\section{Methods}

\section{Study design}

The HEADS study is a two armed pragmatic randomised controlled trial nested in a prospective cohort study (fig $1 \Downarrow$ ). The follow-up study was designed to catch all infants eligible for helmet therapy after a period of paediatric physiotherapy or a single consultation. We invited the parents of eligible infants with moderate to severe skull deformation to include their child in the study at 5 months of age. Participants were randomised
1:1 to either the helmet therapy arm or the natural course arm. Follow-up assessments were performed at 8, 12, and 24 months of age (fig 1). The primary outcome was anthropometric measurement of the skull. A more detailed description of the HEADS study is published elsewhere. ${ }^{36}$

\section{Setting and participants}

Recruitment for the randomised controlled trial was conducted in 29 paediatric physiotherapy practices in the east of the Netherlands between July 2009 and July 2011 by 29 specially trained paediatric physiotherapists. We considered infants to be eligible for the study if they had moderate to severe skull deformation, were aged 5 to 6 months, were born after 36 weeks of gestation, and had no muscular torticollis, craniosynostosis, or dysmorphic features. The course for participating paediatric physiotherapists included detailed information on differentiating between synostotic and non-synostotic skull deformation and between positional and congenital muscular torticollis.

We determined the severity of skull deformation in the transversal plane using the oblique diameter difference index and the cranioproportional index of plagiocephalometry (fig $2 \Downarrow) .{ }^{37}{ }^{38}$ The oblique diameter difference index provides the degree of the plagiocephalic component of skull deformation and is the ratio between the longest cranial diagonal and the shortest cranial diagonal (fig 2) multiplied by $100 \%$, with both located at $40^{\circ}$ from the anterior-posterior line. In addition, we determined the brachycephalic component using the cranioproportional index, which is the ratio between the width and the length of the skull (fig 2).

We included infants in the study if the oblique diameter difference index was $108 \%$ or more or if the cranioproportional index was $95 \%$ or more, or in the case of a mixed form if the oblique diameter difference index was $106 \%$ or more and the cranioproportional index was $92 \%$ or more. Infants with very severe skull deformation were excluded (oblique diameter difference index $>113 \%$ or cranioproportional index $>104 \%$ ). A value of $100 \%$ on the oblique diameter difference index represents a symmetrical head shape. A value of more than $100 \%$ represents asymmetrical skull deformation; the higher the score, the more severe the deformation. A score of $80 \%$ on the cranioproportional index represents an average head shape in Western countries. A higher value represents a larger head width compared with length.

\section{Randomisation and blinding}

At age 5 months, the paediatric physiotherapists selected infants meeting the inclusion criteria for the trial and requested informed consent from the parents. The parents could consult an independent doctor for questions related to trial participation. After inclusion, infants were allocated to their trial arm by computer generated randomisation in blocks of eight. An independent researcher managed the randomisation plan. The research team and parents were blinded for group allocation until the parents had signed the informed consent form and confirmed participation. A researcher (RMW) called the parents and informed them about the randomisation allocation. It was not possible to blind parents during the treatment period. The infant's paediatric physiotherapist, general practitioner, and youth healthcare professional were informed about group allocation after randomisation. Blinded outcome assessments were, however, performed at the 24 month follow-up. 


\section{Intervention and control group}

Parents of infants allocated to the helmet therapy group were asked to make an appointment at one of the four collaborating institutes to obtain a custom-made helmet. Between the institutes two brands of helmets were provided; both helmets used the same mechanism to redirect skull growth. The aim was to start helmet therapy in the infants no later than 6.5 months of age. Parents were instructed to ensure that the helmet was worn for 23 hours a day until their infant was 12 months of age or until satisfactory outcomes were obtained according to both parents and professionals. Parents received additional information on starting the helmet therapy, how the helmet should be worn, cleaning the helmet, and general care. An orthotist regularly monitored the infants for signs of pressure spots, and the helmet was modified or replaced to accommodate skull growth as necessary. The treatment was always supervised by a (paediatric) doctor. During the intervention period, Dutch health insurance companies reimbursed the costs of helmet therapy.

The control group did not receive helmet therapy and natural skull growth was monitored.

\section{Outcome measures}

The paediatric physiotherapists performed measurements at baseline and at age 5, 8, and 12 months. Six of the paediatric physiotherapists who were involved in the study were selected to perform the blinded assessments at 24 months in all infants. These measurements were carried out from February 2011 until March 2013. Every assessment included anthropometric measurement of the skull's shape, a clinical assessment of skull deformation, a motor assessment, and a parental questionnaire. The questionnaire was used to gather information on background characteristics (sex, age, birth rank, health problems, ethnicity, and educational level of the parents) at baseline and parent reported outcomes during all assessments.

\section{Therapy compliance}

The questionnaire administered at 12 months was used to determine whether parents were compliant with the regimen to which their infant was assigned. The questionnaire assessed the age of infants when treatment was discontinued and the reasons for discontinuation. Parents were also asked whether they used additional therapies. Furthermore, the questionnaires at 8 and 12 months also included questions about the fit of the helmet. Originally the protocol specified that compliance would be determined using data from an electronic device built into the helmet to measure compliance with wear. Despite a pilot study, data from the measuring devices proved to be unreliable and we therefore omitted them from further analysis.

\section{Primary outcome Skull shape}

The primary outcome was the anthropometric measurement of the skull's shape at 24 months using the oblique diameter difference index and cranioproportional index. ${ }^{37}{ }^{38} \mathrm{We}$ considered a difference in change score from age 5 to 24 months of 4 oblique diameter difference index points or 5 cranioproportional index points to be relevant between the groups, consistent with one level of severity in skull deformation according to plagiocephalometry criteria (fig 2). Additionally, we report the number of infants who fully recovered, with full recovery defined as an oblique diameter difference index of less than $104 \%$ and a cranioproportional index of less than $90 \%$ (fig 2).

\section{Secondary outcomes Ear deviation}

Severity of ear deviation was expressed by the ear deviation index using plagiocephalometry. The ear deviation index is the ratio between the ear deviation and the length of the skull (fig 2).

\section{Facial asymmetry and occipital lift}

During the clinical assessment the paediatric physiotherapists reported the presence of any facial asymmetry and occipital lift.

\section{Parental satisfaction}

Parental satisfaction with their infant's head shape was assessed in the parental questionnaire using a five point Likert scale, ranging from 1 (not satisfied at all) to 5 (very satisfied).

\section{Motor development}

At baseline, a paediatric physiotherapist assessed the repertoire of gross motor movement using the Alberta infant motor scale, a valid, norm-referenced measurement. We converted raw scores on the scale into standardised $\mathrm{Z}$ scores, using the formula: (individual score-average score)/standard deviation. ${ }^{39}$

To assess motor development at 24 months we used the Bayley scales of infant and toddler development, third edition. ${ }^{40}$ Specially trained physiotherapists administered the test. We converted raw scores to standardised motor composite scores (mean 100 (SD 15)) and scaled scores for fine and gross motor development separately (mean $10(\mathrm{SD}=3))$.

\section{Parental anxiety}

We measured the level of parental state anxiety using the Spielberger state trait anxiety inventory, Dutch version. ${ }^{41}$ The state anxiety scale (20 items) concerns the state of anxiety of parents at a specific moment, and scores range from 20 to 80 ; a higher score represents a higher state of anxiety.

\section{Quality of life}

The infant toddler quality of life questionnaire-short form 47 is a parent reported measure that provides information about the health status and health related quality of life in children aged between 2 months and 5 years. ${ }^{42}$ Since the questionnaire is a "proxy" measure and parental concern might influence outcomes, parent specific scales are included. The questionnaire consists of eight multi-item scales and two single items: the child scales include physical abilities (six items), growth and development (five), bodily pain (two), temperament and moods (six), behaviour (12), general health (six), and change in health (one); the parent scales include parental-impact emotional (four items), parental-impact time (four), and family cohesion (one). Scores for all scales range from 0 to 100 , with a higher score indicating better health.

\section{Side effects}

The questionnaires at 8 and 12 months contained questions about side effects associated with helmet therapy. In consultation with health professionals we defined side effects as skin irritation, pain, sweating, odour of the helmet, problems with accepting the helmet, and feeling hindered in cuddling because of the helmet. Furthermore, in both groups at 8 months parents were asked about the number of hours their baby cried a day and whether their baby had sleep problems. Criteria to define sleep problems in infants are not used consistently in the 
literature ${ }^{43}$; in this study we defined sleep problems as taking more than 20 minutes to fall asleep (daily), or waking more than once every night.

\section{Statistical analyses}

The sample size of the randomised controlled trial of the HEADS study was calculated at 72 (36 in each arm), based on a significance level of $5 \%$, a power of $90 \%$, and a difference in mean improvement of at least 4 (SD 6) oblique diameter difference index points.

We described background and baseline clinical characteristics of the sample for the total group as well as for the intervention and control groups separately; continuous variables with means and standard deviation, and discrete variables with counts and percentages. In a subsequent analysis, we compared the baseline characteristics of the intervention and control groups by means of the independent $t$ test or $\chi^{2}$ test. We determined the representativeness of the randomised controlled trial population by comparing background characteristics and baseline clinical characteristics of the population with eligible non-participants at age 5 months using the independent $t$ test or $\chi^{2}$ test.

For analysis we used two continuous outcome variables (plagiocephaly change score: oblique diameter difference index at age 5 months minus 24 months; and brachycephaly change score: cranioproportional index at age 5 months minus 24 months) and the dichotomous outcome variable (full recovery). Treatment effect was presented as change score in the helmet therapy group minus change score in the natural course group. To test differences in the change scores between the groups, we used analysis of covariance with baseline value (age 5 months) as covariate. Thereafter we carried out multiple regression analyses with baseline values (age 5 months), sex, and parental level of education as covariates. We compared secondary outcomes between groups by means of the independent $t$ test or $\chi^{2}$ test. To analyse the 10 subscales of the infant toddler quality of life questionnaire we performed a multivariate analysis of variance.

We compared the groups on an intention to treat basis. Additionally, we carried out a per protocol analysis. Data analysis was performed using SPSS (version 21.0), and we set the level of significance at 0.05 .

\section{Results \\ Study population}

At the start of this study, the paediatric physiotherapists identified 403 eligible infants (47\% of 866 assessed infants, fig 1). The parents of 84 infants $(21 \%)$ agreed to participate in the trial and those infants were assigned to two groups (42 infants in each group). The main baseline personal and clinical characteristics (sex, age, birth rank, health problems, ethnicity, severity of skull deformation, motor development, and parental satisfaction) did not differ significantly between those who agreed to participate and the infants who were not enrolled, except for the educational level of the parents, which was lower among the participants (see web extra table). The background characteristics of the two trial arms were comparable (table $1 \Downarrow$ ).

A total of 79 infants (94\%) were followed up at the final assessment at 24 months (fig 2). The parents of two infants did not show up for final assessments despite repeated attempts to contact them, two families moved out of the study area, and the parents of one infant could not be contacted.

All infants met the inclusion criteria for either the plagiocephalic component of skull deformation (oblique diameter difference index $\geq 108 \%$ ) or the brachycephalic component (cranioproportional index $\geq 95 \%$ ), or both. The baseline assessments showed statistically significant differences in the shape of the infants' skulls between the two groups (table $2 \Downarrow$ ). Infants in the natural course group presented with more severe plagiocephaly and more often presented with facial asymmetry, whereas infants in the helmet therapy group showed higher brachycephaly scores and more often showed the accompanying occipital lift (table 2).

\section{Therapy allocation and compliance}

After randomisation, seven infants did not start the assigned treatment. Six infants who were allocated to helmet therapy did not start this treatment: in three cases the parents preferred to allow the skull deformity to follow its natural course; in three other cases the doctor advised against helmet therapy.

Additionally, parents of one infant allocated to the natural course arm preferred helmet therapy.

In infants who started in the helmet therapy group, helmet therapy was discontinued at a mean age of 10.0 months (SD 2.0 months, $n=30$ ). Ten of 30 infants received helmet therapy until 12 months of age. The main reasons for parents discontinuing the helmet therapy before 12 months $(n=20)$ was satisfaction with results $(n=8)$, side effects $(n=10)$, dissatisfaction with the results $(n=1)$, and "other" $(n=1)$. Problems with fitting the helmet were reported for 22 of 30 infants $(73 \%)$; the helmet rotated or shifted a few times a week to several times a day. Parents of one infant reported that the helmet came off spontaneously.

Two infants in the natural course group received helmet therapy after the 8 month assessment; the parents were not satisfied with the skull shape.

Three infants in the helmet therapy group and two in the natural course group received additional therapy during the intervention period: manual therapy, osteopathy, or chiropractic.

\section{Primary outcome}

The plagiocephaly change score from age 5 months to 24 months was almost equal for both groups (table $3 \Downarrow$ ): the difference in oblique diameter difference index, calculated as change score in the helmet therapy group minus change score in the natural course group, was -0.2 (95\% confidence interval -1.6 to 1.2 , $\mathrm{P}=0.80$ ). The brachycephaly change score from age 5 months to 24 months was also almost equal for both groups, with a difference in cranioproportional index of $0.2(-1.7$ to 2.2 , $\mathrm{P}=0.81$ ). Additionally, the numbers of infants showing full recovery were comparable in both groups (odds ratio 1.2, 95\% confidence interval 0.4 to 3.3 ).

When adjusting for baseline values, change scores between groups did not differ significantly (adjusted difference in mean plagiocephaly change score $0.9,95 \%$ confidence interval -0.3 to 2.0 and in mean brachycephaly change score $-1.0,-2.5$ to $0.5)$. Adjusting for sex and parental level of education did not alter the treatment effect (plagiocephaly change score: $\beta=1.0$ ( -0.3 to 2.3$), P=0.12$; brachycephaly change score: $\beta=-1.1$ ( -2.8 to 0.5$), \mathrm{P}=0.17$ ).

A per protocol analysis of covariance (helmet therapy $n=34$, natural course $n=45$ ) provided outcomes comparable to the intention to treat analysis (plagiocephaly change score -0.4 , -1.8 to $1.1, \mathrm{P}=0.31$; brachycephaly change score $0.5,-1.5$ to 2.4, $\mathrm{P}=0.11)$. 


\section{Secondary outcomes}

No significant differences were found for the additional clinical outcomes, parent reported outcomes, and motor development. Parents in both arms showed high scores for satisfaction with their infants' skull shape at 24 months (table $4 \Downarrow$ ).

Finally, a multivariate analysis of variance revealed no significant differences between groups for subscales on the infant toddler quality of life questionnaire: Wilks' $\lambda=.826$, $F_{10,63}=1.3, \mathrm{P}=0.24$.

\section{Side effects}

The helmet therapy group had fewer sleep problems (helmet therapy 5/35, 14\%; natural course 10/41, 24\%) and spent fewer hours crying than the natural course group (helmet therapy mean 1.4 (SD 1.2); natural course mean 1.2 (SD 0.9)), although these differences were not significant. In the intervention group all parents $(35 / 35)$ reported one or more side effects related to helmet therapy: problems with acceptance of the helmet $(8 / 33$, $24 \%)$, skin irritation $(32 / 34,96 \%)$, augmented sweating (24/34, $71 \%)$, unpleasant odour of the helmet $(25 / 33,76 \%)$, pain associated with the helmet $(9 / 27,33 \%)$, and feeling hindered from cuddling their child $(24 / 31,77 \%)$.

\section{Discussion}

This pragmatic randomised controlled trial found no evidence of a significant or clinically meaningful difference in improvement of skull shape at 2 years of age between infants who were treated with helmet therapy and those in whom the natural course of skull deformation was awaited. Despite improvement in skull shape in both groups, only a quarter of the participants showed full recovery. Overall, parents were satisfied to very satisfied with the recovery of their infants' skull deformation at 2 years old. However, the parents of infants who were treated with a helmet showed slightly higher satisfaction scores and a slightly lower state of anxiety when their infants were 2 years of age.

Helmet therapy did not influence the infants' motor development, quality of life, sleeping, or crying. Side effects of helmet therapy were reported by all parents.

\section{Strengths and limitations of this study}

Strengths of this study include the randomised allocation of treatment, nested design, high follow-up rates, use of various long term outcomes measures, and both plagiocephaly and brachycephaly being studied.

The HEADS trial is the first study to provide evidence from a randomised controlled trial on the long term effectiveness of helmet therapy for skull deformation. The nested design enables us to determine the generalizability of study outcomes. As well as having anthropometric outcome measures, this study presented parent reported outcomes (for example, subjective assessments and quality of life assessment) and side effects. A high follow-up rate of $94 \%$ ensures the power of the study and indicates that the follow-up was not selective.

The use of two outcome measures (oblique diameter difference index and analysis of covariance) could be disputed. However, positional skull deformation usually presents with components of both brachycephaly and plagiocephaly and not just as one type or the other. ${ }^{2}$ Moreover, helmets are prescribed for all variations of moderate to severe positional skull deformation, so we therefore included both components of skull deformation.
Limitations of this study include the difference of severity of skull deformation at baseline between both arms of the trial, a low participation rate, limited generalisability of study results to specific subgroups of infants, and no assessment of daily wearing time of the helmet.

Despite between group differences in the baseline clinical characteristics at randomization, this was tackled using the planned analysis of covariance for the comparison of mean differences. The improvement assessed by anthropometric measurements showed no differences at 24 months, yet the parents of infants in the helmet therapy group showed slightly higher satisfaction scores and lower anxiety levels. This might be explained by the fact that simply offering treatment may reassure parents.

The parents of $21 \%$ of eligible infants agreed to participate in the trial. Participating parents had a lower level of education than non-participating parents. It has been described before that parents with a higher level of education might have stronger preferences for treatment and are thereby less likely to agree with randomisation. ${ }^{44}$

Another limitation of the study is that the results concern infants with moderate to severe skull deformation and therefore are not generalisable to cases of very severe skull deformation. We decided to exclude very severe cases, since we expected selection bias on the basis of severity of the deformation and selective loss to follow-up in infants with very severe skull deformation who would have been allocated to the natural course. Eventually, only 29/432 (7\%) of infants who were eligible for helmet therapy at age 5 months were excluded on the basis the severity of skull deformation. Results are also not generalisable to infants with an underlying congenital condition or muscular torticolllis, or infants who were born preterm. Inclusion of infants born preterm would have complicated the treatment protocol and the interpretation of outcomes. The prevalence of positional skull deformation in infants born preterm is high, but the natural course seems favourable. ${ }^{46}$ Additionally, in infants born preterm the corrected age has to be used for the start of treatment and outcome measurements, which would have complicated interpretation and the generalisability of study outcomes.

A final limitation is that we were not able to study the exact wear time of the helmet. However, in this pragmatic study we wanted to study the effect of helmet therapy in routine everyday practice, including parent instructions and regular check-ups to monitor treatment and assess improvements in skull shape.

\section{Strengths and weaknesses in relation to other studies}

The various literature reviews suggest that helmet therapy may be more effective in correcting skull deformation in infants aged 6 months than other conservative treatments, but urge the need for evidence from randomised controlled trials. ${ }^{26} 28343547$

Contrary to the present study, a recent study advocated the use of a helmet for moderate to severe skull deformation. Both this and our study had an equal intervention period: therapy started at 6 months of age and ended at 10 months on average. However, the recent study was a non-randomised study with no blinded assessors, using different time intervals of follow-up and a non-validated outcome measure. ${ }^{30}$

Previous studies often did not comprise long term outcomes and systematic assessment of side effects. One retrospective study described how $22.4 \%$ of infants experienced side effects of helmet therapy. This is in contrast with our study, in which side effects were reported in all infants, probably because we 
used a broader definition of side effects and side effects were self reported by the parents. ${ }^{48}$ Additionally, the clinical meaning of the effects of helmet therapy presented in previous studies can be disputed. ${ }^{34}$ In the present study we defined a clinically meaningful difference as 4 oblique diameter difference index points or 5 cranioproportional index points, consistent with one level of severity in skull deformation according to plagiocephalometry criteria (fig 2). We chose this cut-off point based on expert opinion, and it should represent a difference that is clinically visible. Both our randomised study design and its focus on clinically meaningful differences, rather than just significant differences, are strengths of this study compared with previous studies.

In both arms of this study, brachycephaly showed a more favourable course of recovery than plagiocephaly. A cohort study of 129 infants with skull deformation at age 6 months showed comparable results at age 4 years when the natural course was awaited: the improvement in plagiocephaly (oblique cranial length ratio at age 6 months: 108.6 (SD 3.3); age 4 years: 105.4 (SD 2.6)) is almost equal to the mean change score at age 5 months to 24 months of the natural course group (oblique diameter difference index 3.1 (SD 3.3)) in the present study. The improvement of brachycephaly (cephalic index at age 6 months: 92.6 (SD 6.6); age 4 years: 87.0 (SD 4.7)) is also in line with findings in the natural course group in the present study (change score on cranioproportional index from 5 months to 24 months 6.8 (SD 4.4)). ${ }^{21}$

Finally, interpreting parent reported outcomes can be difficult. As in other studies, the objective outcomes in this study did not match the subjective assessments. ${ }^{27} 4950$ Non-clinical factors may be as important as clinical factors in assessments of satisfaction. ${ }^{51}$

\section{Practice implications}

This study indicates that helmet therapy has no added value in the treatment of moderate to severe skull deformation in healthy infants. A cost study performed in both arms of the present study, parallel to the HEADS effectiveness study, showed that the total costs per infant treated with a helmet were substantially higher ( $\mathrm{n}=20, € 1401 ; £ 1157 ; \$ 1935)$ than for infants in whom the natural course of skull deformation was awaited $(n=14$, $€ 157){ }^{52}$ Based on the equal effectiveness of helmet therapy compared with the natural course, the high prevalence of side effects and the high costs of treatment, we discourage the use of helmet therapy as a standard treatment for healthy infants with moderate to severe skull deformation. Outcomes are expected to hold for all types of custom-made helmets comprising a rigid plastic shell with a foam lining that are designed to fit snugly over the infant's head and leaving room for skull growth at the flattened area.

This conclusion is therefore likely to affect decisions of parents, policymakers, insurance companies, and a wide range of clinicians such as paediatricians, general practitioners, youth healthcare professionals, paediatric physiotherapists, orthotists, paediatric neurosurgeons, and craniofacial surgeons internationally.

Our study also indicated that $75 \%$ of infants continued to have some degree of skull deformation at 2 years of age, mainly the plagiocephalic component. Skull deformation does not completely resolve in all cases by natural course, and helmet therapy does not seem to have an added value for recovery. Therefore we emphasise the importance of prevention, early detection, and early treatment with paediatric physiotherapy of skull deformation. ${ }^{22}{ }^{35}$ Additionally, our cut-off points for normal head shape might be rigid in comparison with others. ${ }^{253}$ Therefore, the $75 \%$ of infants with persisting skull deformation in the present study could be an overestimation of the prevalence of the condition at an older age. It remains arguable what an acceptable head shape is in young infants and at an older age when the head is covered with hair.

\section{Unanswered questions and future research}

This is the first randomised controlled trial on helmet therapy in infants with positional skull deformation. Although we conclude no significant difference, this study was not powered for equivalence. Ideally, the study should be repeated with an adequate sample size to confirm the non-inferiority of helmet therapy. However, we question whether aiming for another randomised controlled trial will be realistic since helmet therapy is not reimbursed in most countries. Results from the HEADS non-randomised controlled trial will be presented in the near future and might provide additional evidence to set next to the results of the present randomised controlled trial. Future research should determine the effects of helmet therapy in very severe skull deformation.

We thank the parents and infants for participating in the HEADS study; the paediatric physiotherapists for recruiting participants and acquiring the data; the orthotists and (paediatric) doctors, who provided helmet therapy, for their willingness to cooperate with the HEADS study; Henk Wierenga, for his role as independent adviser to parents; youth healthcare professionals in the study area for supporting the HEADS study; Marjon Rouwette-Witting and Jorien Pierik for managing the randomisation plan; Carine Doggen for reading and editing the manuscript before submission; and the HEADS project group, HEADS project group advisors, TNO Child Health, and the Royal Dutch Society for Physical Therapy for their collaboration.

Contributors: RMW was responsible for the day to day management of the trial, development of the trial, data collection, and the first draft of the manuscript. MMB, CGMG, CPBP, LAV, and MJIJ conceived and designed the HEADS study and obtained funding. MMB, LAV, and MJIJ supervised the study. LAV and RMW trained the trial assessors (paediatric physiotherapists). RWM and CGMG performed the data analysis. All authors interpreted the data and critically revised the manuscript for important intellectual content, had full access to all of the data in the study, and can take responsibility for the integrity of the data and the accuracy of the data analysis. MMB is the guarantor.

Funding: This study was funded by ZonMw, the Netherlands Organization for Health Research and Development (grant No 170.992.501). Besides the initial review process before funding and amendments, ZonMw had no involvement in the study design, management of the study, data analysis, writing, and publications. All researcher activities were independent of the funding source.

Competing interests: All authors have completed the ICMJE uniform disclosure form at www.icmje.org/coi_disclosure.pdf and declare: no support from any organisation for the submitted work; no financial relationships with any organisations that might have an interest in the submitted work in the previous three years; no other relationships or activities that could appear to have influenced the submitted work.

Ethical approval: This study was approved by the medical ethics committee of Medisch Spectrum Twente, hospital of Enschede, Netherlands, 8 January 2009 (reference NL24352.044.08).

Data sharing: Relevant patient level data are available from the corresponding author on reasonable request. Informed consent for data sharing was not obtained but the presented data are anonymised.

Transparency: The manuscript's guarantor affirms that this manuscript is an honest, accurate, and transparent account of the study being reported; that no important aspects of the study have been omitted; and 


\section{What is already known on this topic}

Positional skull deformation is common in infancy

Helmet therapy is often prescribed in infants with positional skull deformation

Evidence from randomised controlled trials for the effectiveness of helmet therapy compared with the natural course of the condition is lacking

\section{What this study adds}

No effect of helmet therapy can be shown in infants with moderate to severe positional skull deformation

All parents reported side effects; however, helmet therapy did not influence infants' motor development or health related quality of life

that any discrepancies from the study as planned (and, if relevant, registered) have been explained.

Boere-Boonekamp MM, van der Linden-Kuiper LT. Positional preference: prevalence in infants and follow-up after two years. Pediatrics 2001;107:339-43.

2 Meyer-Marcotty P, Bohm H, Linz C, Kochel J, Blecher C, Keil N, et al. Spectrum of positional deformities-is there a real difference between plagiocephaly and brachycephaly? J Craniomaxillofac Surg 2014 (in press).

3 Argenta L, David L, Thompson J. Clinical classification of positional plagiocephaly. J Craniofac Surg 2004;15:368-72.

4 Clarren SK. Plagiocephaly and torticollis: etiology, natural history, and helmet treatment. $J$ Pediatr 1981;98:92-5.

5 Mulliken JB, Vander Woude DL, Hansen M, LaBrie RA, Scott RM. Analysis of posterior plagiocephaly: deformational versus synostotic. Plast Reconstr Surg 1999;103:371-80.

6 Collett BR, Gray KE, Starr JR, Heike CL, Cunningham ML, Speltz ML. Development at age 36 months in children with deformational plagiocephaly. Pediatrics 2013;131:e109-15

7 Hutchison BL, Stewart AW, Mitchell EA. Characteristics, head shape measurements and developmental delay in 287 consecutive infants attending a plagiocephaly clinic. Acta Paediatr 2009;98:1494-9.

8 Kennedy E, Majnemer A, Farmer JP, Barr RG, Platt RW. Motor development of infants with positional plagiocephaly. Phys Occup Ther Pediatr 2009;29:222-35.

9 Kordestani RK, Patel S, Bard DE, Gurwitch R, Panchal J. Neurodevelopment delays in children with deformational plagiocephaly. Plast Reconstr Surg 2006;117:207-18.

10 Collett B, Breiger D, King D, Cunningham M, Speltz M. Neurodevelopmental implications of "deformational" plagiocephaly. J Dev Behav Pediatr 2005;26:379-89.

11 Steinbok P, Lam D, Singh S, Mortenson PA, Singhal A. Long-term outcome of infants with positional occipital plagiocephaly. Childs Nerv Syst 2007;23:1275-83.

12 Argenta LC, David LR, Wilson JA, Bell WO. An increase in infant cranial deformity with supine sleeping position. J Craniofac Surg 1996;7:5-11.

13 De Jonge GA, Engelberts AC, Koomen-Liefting AJ, Kostense PJ. Cot death and prone sleeping position in The Netherlands. BMJ 1989;298:722.

14 Dwyer T, Ponsonby AL, Blizzard L, Newman NM, Cochrane JA. The contribution of changes in the prevalence of prone sleeping position to the decline in sudden infant death syndrome in Tasmania. JAMA 1995;273:783-9.

15 Fleming PJ, Gilbert R, Azaz Y, Berry PJ, Rudd PT, Stewart A, et al. Interaction between bedding and sleeping position in the sudden infant death syndrome: a population based case-control study. BMJ 1990;301:85-9.

16 Kane AA, Mitchell LE, Craven KP, Marsh JL. Observations on a recent increase in plagiocephaly without synostosis. Pediatrics 1996;97(6 Pt 1):877-85.

17 Turk AE, McCarthy JG, Thorne $\mathrm{CH}$, Wisoff JH. The "back to sleep campaign" and deformational plagiocephaly: is there cause for concern? J Craniofac Surg 1996;7:12-8.

18 American Academy of Pediatrics AAP Task Force on Infant Positioning and SIDS: positioning and SIDS. Pediatrics 1992;89(6 Pt 1):1120-6.

19 Hutchison BL, Hutchison LA, Thompson JM, Mitchell EA. Plagiocephaly and brachycephaly in the first two years of life: a prospective cohort study. Pediatrics 2004;114:970-80.

20 Van Vlimmeren LA, van der Graaf Y, Boere-Boonekamp MM, L'Hoir MP, Helders PJM, Engelbert RHH. Risk factors for deformational plagiocephaly at birth and at 7 weeks of age: a prospective cohort study. Pediatrics 2007;119:e408-18

21 Hutchison BL, Stewart AW, Mitchell EA. Deformational plagiocephaly: a follow-up of head shape, parental concern and neurodevelopment at ages 3 and 4 years. Arch Dis Child 2010;96:85-90.

22 Van Vlimmeren LA, van der Graaf Y, Boere-Boonekamp MM, L'Hoir MP, Helders PJ, Engelbert RH. Effect of pediatric physical therapy on deformational plagiocephaly in children with positional preference: a randomized controlled trial. Arch Pediatr Adolesc Med 2008;162:712-8.

23 Nederlands Centrum Jeugdgezondheid. JGZ-richtlijn preventie, signalering en aanpak van voorkeurshouding en schedelvervorming. TNO innovation for life, 2012.

24 Cabrera-Martos I, Valenza MC, Benitez-Feliponi A, Robles-Vizcaino C, Ruiz-Extremera A, Valenza-Demet G. Clinical profile and evolution of infants with deformational plagiocephaly included in a conservative treatment program. Childs Nerv Syst 2013;29:1893-8

25 Persing J, James H, Swanson J, Kattwinkel J; American Academy of Pediatrics Committee on Practice and Ambulatory Medicine, Section on Plastic Surgery and Section on Neurological Surgery.. Prevention and management of positional skull deformities in infants. Pediatrics 2003;112:199-202.

26 Flannery AB, Looman WS, Kemper K. Evidence-based care of the child with deformational plagiocephaly, part II: management. J Pediatr Health Care 2012;26:320-31.

27 Kluba S, Kraut W, Reinert S, Krimmel M. What is the optimal time to start helmet therapy in positional plagiocephaly? Plast Reconstr Surg 2011;128:492-8.

28 Xia JJ, Kennedy KA, Teichgraeber JF, Wu KQ, Baumgartner JB, Gateno J. Nonsurgical treatment of deformational plagiocephaly: a systematic review. Arch Pediatr Adolesc Med 2008;162:719-27.

29 Statistics Netherlands. Population; sex, age, origin and generation, 1 January. Den Haag/Heerlen Statistics Netherlands, 2013.
30 Kluba S, Kraut W, Calgeer B, Reinert S, Krimmel M. Treatment of positional plagiocephaly - helmet or no helmet? J Craniomaxillofac Surg 2014 (in press)

31 Lipira AB, Gordon S, Darvann TA, Hermann NV, Van Pelt AE, Naidoo SD, et al. Helmet versus active repositioning for plagiocephaly: a three-dimensional analysis. Pediatrics 2010;126:e936-45

32 Loveday BP, de Chalain TB. Active counterpositioning or orthotic device to treat positional plagiocephaly? J Craniofac Surg 2001;12:308-13.

33 Vles JS, Colla C, Weber JW, Beuls E, Wilmink J, Kingma H. Helmet versus nonhelmet treatment in nonsynostotic positional posterior plagiocephaly. J Craniofac Surg 2000;11:572-4.

34 Goh JL, Bauer DF, Durham SR, Stotland MA. Orthotic (helmet) therapy in the treatment of plagiocephaly. Neurosurg Focus 2013;35:E2.

35 Robinson S, Proctor M. Diagnosis and management of deformational plagiocephaly. $J$ Neurosurg Pediatr 2009:3:284-95.

36 Van Wijk RM, Boere-Boonekamp MM, Groothuis-Oudshoorn CG, van Vlimmeren LA, IJzerman MJ. HEImet therapy Assessment in infants with Deformed Skulls (HEADS): protocol for a randomised controlled trial. Trials 2012;13:108.

37 Van Adrichem LNA, van Vlimmeren LA, Cadanova D, Helders PJM, Engelbert RHH, van Neck HJW, et al. Validation of a simple method for measuring cranial deformities (plagiocephalometry). J Craniofac Surg 2008;19:15-21.

38 Van Vlimmeren LA, Takken T, van Adrichem LN, van der Graaf Y, Helders PJ, Engelbert RH. Plagiocephalometry: a non-invasive method to quantify asymmetry of the skull; a reliability study. Eur J Pediatr 2006;165:149-57.

39 Piper MC, Pinnell LE, Darrah J, Maguire T, Byrne PJ. Construction and validation of the Alberta Infant Motor Scale (AIMS). Can J Public Health 1992;83(Suppl 2):S46-50.

40 Bayley N. Manual for the Bayley scales of infant and toddler development.3rd edn. Psychological Corporation, 2006

41 Van der Ploeg HM, Defares PB, Spielberger CD. Handleiding bij de Zelf-Beoordelings Vragenlijst, ZBV: Een Nederlandstalige bewerking van de Spielberger state-trait anxiety inventory. Swets \& Zeitlinger, 1980.

42 Landgraf JM, Vogel I, Oostenbrink R, van Baar ME, Raat H. Parent-reported health outcomes in infants/toddlers: measurement properties and clinical validity of the ITQOL-SF47. Qual Life Res 2013;22:635-46.

43 Mindell JA, Kuhn B, Lewin DS, Meltzer LJ, Sadeh A. Behavioral treatment of bedtime problems and night wakings in infants and young children. Sleep 2006;29:1263-76.

44 Harth SC, Thong YH. Sociodemographic and motivational characteristics of parents who volunteer their children for clinical research: a controlled study. BMJ 1990;300:1372-5

45 Rovers MM, Straatman H, Ingels K, van der Wilt GJ, van den Broek P, Zielhuis GA. Generalizability of trial results based on randomized versus nonrandomized allocation of OME infants to ventilation tubes or watchful waiting. J Clin Epidemiol 2001;54:789-94.

46 Nuysink J, Eijsermans MJ, van Haastert IC, Koopman-Esseboom C, Helders PJ, de Vries $\mathrm{LS}$, et al. Clinical course of asymmetric motor performance and deformational plagiocephaly in very preterm infants. J Pediatr 2013;163:658-65 e1.

47 Bialocerkowski AE, Vladusic SL, Howell SM. Conservative interventions for positional plagiocephaly: a systematic review. Dev Med Child Neurol 2005;47:563-70.

48 Wilbrand JF, Wilbrand M, Malik CY, Howaldt HP, Streckbein P, Schaaf H, et al. Complications in helmet therapy. J Craniomaxillofac Surg 2012;40:341-6.

49 Feijen M, Schuckman M, Habets E, van der Hulst R. Positional plagiocephaly and brachycephaly: is there a correlation between subjective and objective assessment of cranial shape? J Craniofac Surg 2012;23:998-1001.

50 Katzel EB, Koltz PF, Sbitany H, Emerson C, Girotto JA. Real versus perceived improvements of helmet molding therapy for the treatment of plagiocephaly. Plast Reconstr Surg 2010;126:19e-21e.

51 Suarez-Almazor ME, Conner-Spady B, Kendall CJ, Russell AS, Skeith K. Lack of congruence in the ratings of patients' health status by patients and their physicians. Med Decis Making 2001:21:113-21.

52 Van der Ploeg CBP, Van Wijk RM, van den Akker EM, Boere-Boonekamp MM. Cost assessment of helmet treatment compared to natural course in infants with skull deformation. TNO, 2013

53 Hutchison BL, Hutchison LA, Thompson JM, Mitchell EA. Quantification of plagiocephaly and brachycephaly in infants using a digital photographic technique. Cleft Palate Craniofac J 2005;42:539-47.

Accepted: 29 March 2014

\section{Cite this as: BMJ 2014;348:g2741}

This is an Open Access article distributed in accordance with the Creative Commons Attribution Non Commercial (CC BY-NC 3.0) license, which permits others to distribute, remix, adapt, build upon this work non-commercially, and license their derivative works on different terms, provided the original work is properly cited and the use is non-commercial. See: http://creativecommons.org/licenses/by-nc/3.0/. 


\section{Tables}

Table 1 | Characteristics of study population of infants with skull deformation. Values are numbers (percentages)* unless stated otherwise

\begin{tabular}{lccc} 
Characteristics & Total population $(\mathbf{n}=\mathbf{8 4})$ & Helmet therapy $(\mathbf{n}=\mathbf{4 2})$ & Natural course $(\mathbf{n}=\mathbf{4 2})$ \\
Boys & $61 / 84(73)$ & $32 / 42(76)$ & $29 / 42(69)$ \\
\hline Mean (SD) age at baseline (months) & $5.1(0.4)$ & $5.1(0.4)$ & $5.1(0.3)$ \\
\hline Mean (SD) age at follow-up (months) & $24.8(4.4)$ & $25.0(3.5)$ & $24.6(5.1)$ \\
\hline Birth rank (first born) & $39 / 79(49)$ & $20 / 40(50)$ & $19 / 39(49)$ \\
\hline Health problems $\dagger$ & $6 / 84(7)$ & $4 / 42(10)$ & $2 / 40(5)$ \\
\hline Ethnicity (ethnic minority) $\neq$ & $5 / 77(7)$ & $4 / 41(10)$ & $1 / 36(3)$ \\
\hline Education level of parents§: & & & $9 / 39(23)$ \\
\hline Low & $24 / 81(30)$ & $15 / 42(36)$ & $19 / 39(49)$ \\
\hline Medium & $34 / 81(42)$ & $15 / 42(36)$ & $11 / 39(28)$ \\
\hline High & $23 / 81(28)$ & $12 / 42(29)$ & \\
\hline
\end{tabular}

Numbers may not add up to group totals because of missing data.

*Groups compared using $t$ test or $\chi^{2}$ test.

†Problems with sight, hearing, oesophageal reflux, developmental dysplasia of hip, congenital heart disease, or inguinal hernia.

$\ddagger$ At least one parent born outside of the Netherlands.

§Low education level: lower technical and vocational training and lower general secondary education; medium education level: intermediate vocational training and advanced secondary education; and high education level: higher vocational education and university. Percentages may not total $100 \%$, due to rounding off. 
Table 2| Baseline clinical characteristics of study population at age 5 months. Values are means (standard deviations) unless stated otherwise*

\begin{tabular}{lcc} 
Clinical characteristics & Helmet therapy $(\mathbf{n = 4 2 )}$ & Natural course $(\mathbf{n = 4 2 )}$ \\
Plagiocephaly (ODDI) $\dagger$ & $107.2(3.9)$ & $109.2(2.9)$ \\
\hline Brachycephaly (CPI) $\dagger$ & $93.4(6.9)$ & $90.3(6.2)$ \\
\hline Ear deviation (EDI) & $4.7(3.5)$ & $5.5(3.0)$ \\
\hline No (\%) with facial asymmetry & $7(17)$ & $13(31)$ \\
\hline No (\%) with occipital lift & $18(43)$ & $10(24)$ \\
\hline Motor development (AIMS Z-score) & $-0.7(1.0)$ & $-0.7(1.0)$ \\
\hline Parental satisfaction & $2.8(0.8)$ & $3.0(1.0)$ \\
\hline State anxiety (STAI-DY) & $30.8(7.1)$ & $32.2(8.6)$ \\
\hline
\end{tabular}

ODDI=oblique diameter difference index (value of $100 \%$ represents purely symmetrical head shape, value $>100$ represents asymmetrical skull deformation; the higher the score, the more severe the deformation); $\mathrm{CPI}=$ cranioproportional index (score of $80 \%$ represents an average head shape in Western countries, higher value represents a larger head width compared with length); $\mathrm{EDI}=$ ear deviation index (value of 0 represents no ear deviation; the higher the score above 0 , the more severe the ear deviation). AIMS=Alberta infant motor scale standardised Z scores (individual score minus average score divided by standard deviation); STAI-DY=Dutch version of Spielberger state-trait anxiety inventory (scores range from 20 to 80; a higher score represents a higher state anxiety). *Groups compared using $t$ test or $\chi^{2}$ test. $\dagger P<0.05$. 
Table 3| Primary outcomes, measured at 24 months. Values are means (standard deviations) unless stated otherwise

ANCOVA; adjusted means $(95 \% \mathrm{Cl}) \dagger$

Outcomes

Helmet therapy $(n=39)$ Natural course $(n=40) \quad P$ value* Helmet therapy Natural course P value

Plagiocephaly change scoreł

$2.9(2.9) \quad 3.1(3.3)$

Brachycephaly change score§

$7.0(4.1)$

$6.8(4.4)$

$0.80 \quad 3.4$ (2.6 to 4.2$) \quad 2.6$ (1.8 to 3.4$)$

0.13

No (\%) with full recovery

$10(26)$

$9(23)$

$0.81 \quad 6.4(5.3$ to 7.5$) \quad 7.4(6.4$ to 8.5$) \quad 0.20$

ANCOVA=analysis of covariance.

${ }^{*}$ Groups compared using $t$ test or $\chi^{2}$ test.

†ANCOVA model with baseline measurement at age 5 months as covariate.

‡Oblique diameter difference index at age 5 months minus at age 24 months.

$\S$ Cranioproportional index at age 5 months minus at age 24 months.

TOblique diameter difference index $<104 \%$ and cranioproportional index $<90 \%$.

${ }^{* *}$ Not analysed because of low number of cases. 
Table 4 | Secondary outcomes, measured at age 24 months. Values are means (standard deviations) unless stated otherwise

\begin{tabular}{|c|c|c|c|c|}
\hline Secondary outcomes & Helmet therapy $(n=39)$ & Natural course $(n=40)$ & P value ${ }^{*}$ & Helmet therapy-natural course \\
\hline Ear deviation change score $(\mathrm{n}=79) \dagger$ & $2.0(4.0)$ & $1.9(3.6)$ & 0.86 & $0.2(-1.5$ to 1.8$)$ \\
\hline No (\%) with facial asymmetry & $5 / 38(13)$ & 10/39 (26) & 0.17 & $0.4(0.1$ to 1.4$) \ddagger$ \\
\hline No (\%) with occipital lift & $4 / 38(11)$ & $2 / 40(5)$ & 0.36 & $2.2(0.4$ to 13.0$) \ddagger$ \\
\hline Parental satisfaction $(n=77)$ & $4.6(0.5)$ & $4.4(0,6)$ & 0.06 & $0.2(-0.1$ to 0.5$)$ \\
\hline State anxiety (STAI-DY) $(\mathrm{n}=76)$ & $27.4(6.5)$ & $31.3(9.2)$ & 0.04 & $-3.9(-7.5$ to -0.2$)$ \\
\hline BSID-III composite score $(n=77)$ & $97.2(9.4)$ & $99.0(11.6)$ & 0.17 & $-1.8(-6.6$ to 3.0$)$ \\
\hline BSID-III fine scale $(n=78)$ & $10.0(1.9)$ & $10.8(2.2)$ & 0.21 & $-0.7(-1.7$ to 0.2$)$ \\
\hline BSID-III gross scale $(n=78)$ & $9.1(1.8)$ & $8.8(2.4)$ & 0.58 & $0.2(-0.7$ to 1.2$)$ \\
\hline
\end{tabular}

STAI-DY=Dutch version of Spielberger state-trait anxiety inventory (scores range from 20 to 80; a higher score represents a higher state anxiety); BSID III=Bayley scales of infant and toddler development, third edition (standardised motor composite scores mean 100 (SD 15), scaled scores for fine and gross motor development mean 10 (SD 3).

*Groups compared using $t$ test or $x^{2}$ test.

†Ear deviation index measurement at age 5 months minus at age 24 months.

‡Odds ratio (95\% confidence interval). 


\section{Figures}

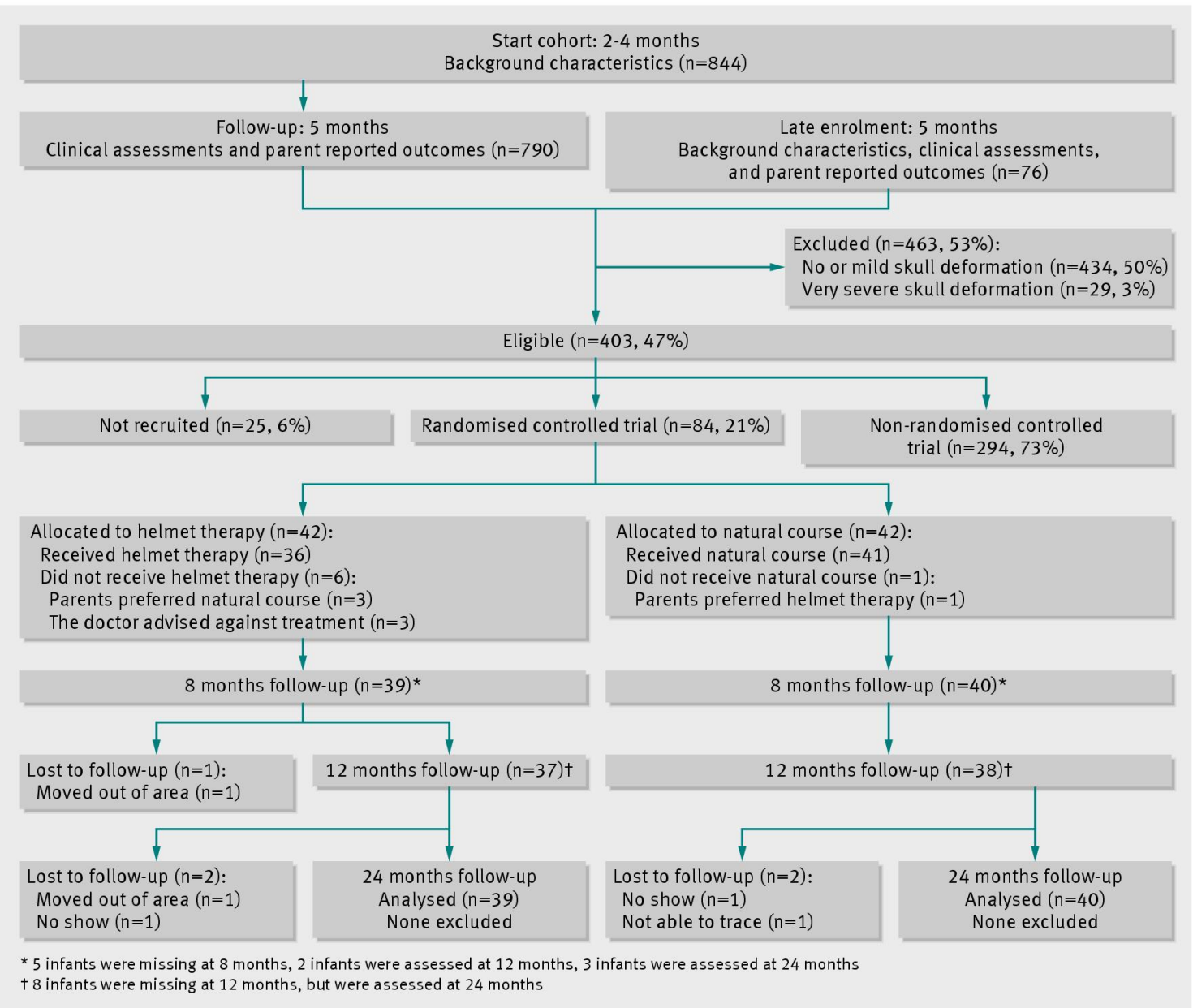

Fig 1 Flowchart of HElmet therapy Assessment in Deformed Skulls study 


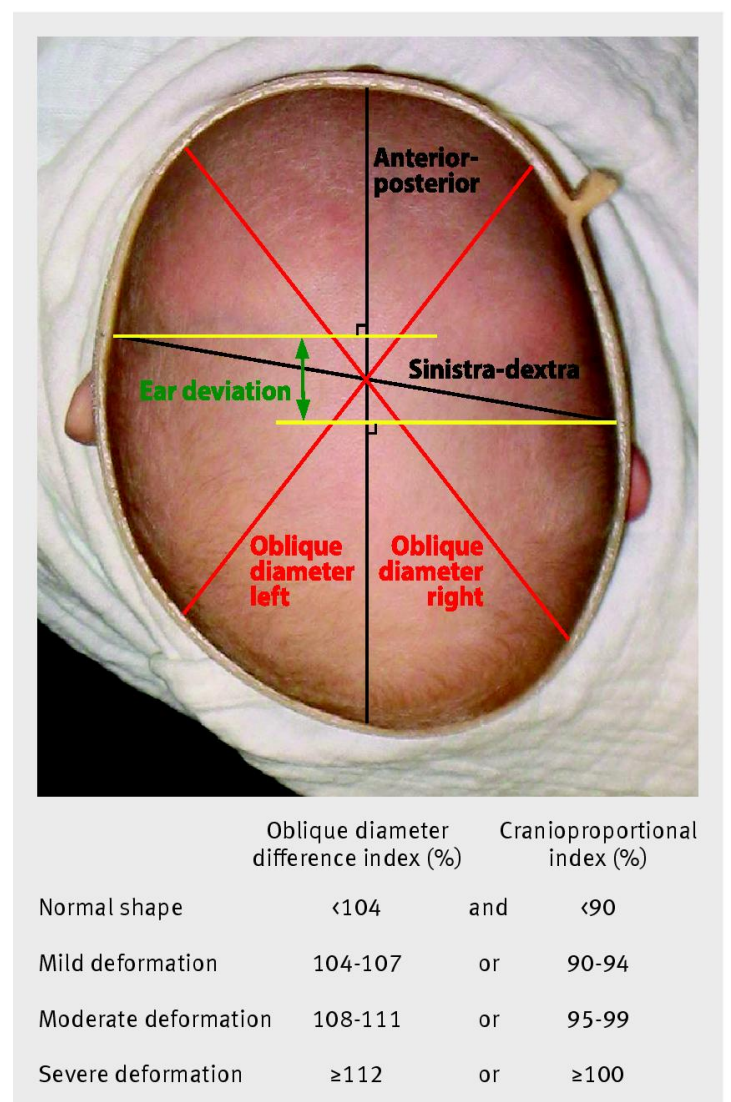

Fig 2 Plagiocephalometry and cut-off points for severity of skull deformation. Illustration shows left occipital flattening of skull and thermoplastic measuring ring with digitally drawn lines used in plagiocephalometry. Indices were calculated by the following formulas: cranioproportional index, sinistra-dextra/anterior-posterior $\times 100 \%$; oblique diameter difference index, longest oblique diameter/shortest oblique diameter $\times 100 \%$; and ear deviation index, ear deviation/anterior-posterior $\times 100 \%$ 